\title{
New paths to beating bacteria
}

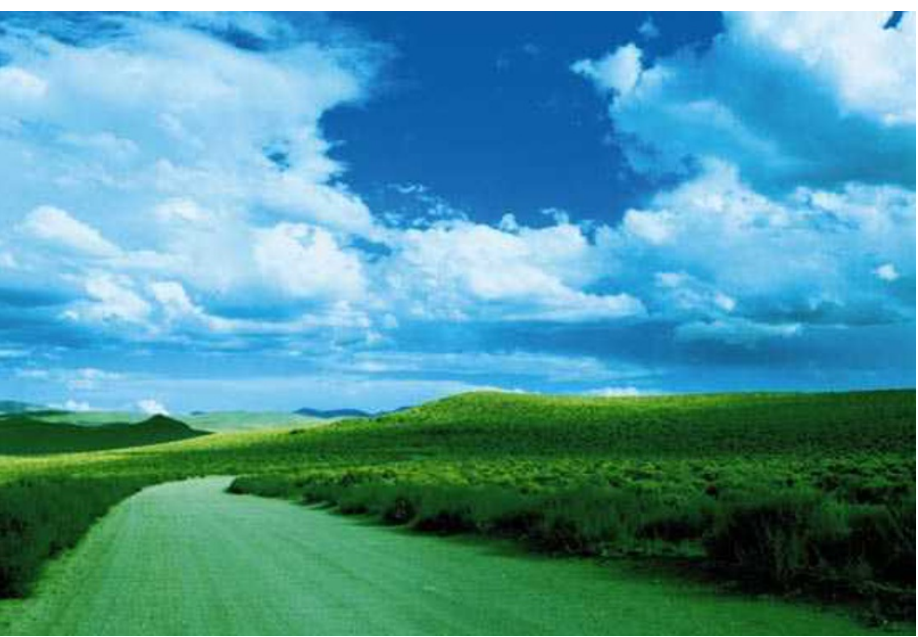

The need for new drugs to combat multidrug-resistant bacteria remains pressing. Now, two recent papers published in Science describe novel agents that target different mechanisms to currently approved antibacterials, which may help tackle this challenge.

The first study, by Haydon and colleagues, set out to target bacterial cell division. FtsZ is an essential bacterial protein that undergoes GTP-dependent polymerization to form the $\mathrm{Z}$ ring — the site of cell division - promoting the sequential recruitment of additional proteins that are vital to the process. Of these proteins, FtsZ is considered to be a particularly attractive target, as it is a distant relative of mammalian tubulin - the target of anticancer drugs such as paclitaxel - suggesting that it might also be amenable to inhibitor development.

Indeed, previous studies have identified several compounds that can inhibit FtsZ activity in vitro, but so far, none have demonstrated in vivo efficacy. However, using one such compound (3-methoxybenzamide) as a starting point, Haydon and colleagues used a fragmentbased approach to develop the more potent cell-division inhibitor PC190723. Mechanistic studies revealed that PC190723 directly inhibited the GTPase activity of FtsZ, and bound to a region of the protein analogous to the paclitaxel-binding site of human tubulin.

In vitro, PC190723 exhibited strong antibacterial activity against Bacillus subtilis, as well as all strains and species of staphylococci tested. Notably, this included both a methicillin-resistant as well as a multidrug-resistant strain of Staphylococcus aureus. Next, to determine in vivo efficacy, mice were treated with a single subcutaneous or intravenous dose of PC190723 1 hour following inoculation with a potentially lethal dose of S. aureus. Remarkably, all mice survived - in stark contrast to the death of all controls.

In the second paper, Rasko and colleagues pursued an alternative strategy of inhibiting microbial virulence rather than growth, which is thought to present less selective pressure and therefore reduce the likelihood of resistance developing. To do this, they aimed to target QseC, a conserved membrane histidine sensor kinase. By sensing both host adrenergic signalling molecules and bacterial aromatic signal autoinducer 3, QseC autophosphorylates, which triggers the subsequent phosphorylation of the transcription factor QseB to promote the transcription of key virulence genes.
By screening an extensive library of small organic molecules and performing subsequent structureactivity modifications, Rasko and colleagues identified the potent QseC inhibitor, LED209, which they then tested against a variety of bacteria.

In enterohaemorrhagic Escherichia coli, LED209 inhibited QseC signal binding, thereby inhibiting downstream virulence gene expression. Oral LED209 treatment similarly inhibited Salmonella typhimurium virulence gene expression in mice, when given before and after infection, with $80 \%$ of treated mice remaining alive after 24 hours and $20 \%$ surviving up to 12 days. By contrast, all control mice died within 72 hours. Strikingly, a single oral dose of LED209 protected mice that had already been exposed to Francisella tularensis, with $80 \%$ of mice still living after 9 days compared with just $10 \%$ of controls.

Overall, these studies highlight the potential of two underexploited antibacterial targets, as well as identify compounds with promising properties to be optimized into candidate drugs active against bacterial strains that represent a significant public health threat.

Sarah Crunkhorn

ORIGINAL RESEARCH PAPERS Haydon, D. J. et al. An inhibitor of $\mathrm{FtsZ}$ with potent and selective anti-staphylococcal activity. Science 321, 1673-1675 (2008) | Rasko, D. A. et al. Targeting QseC signaling and virulence for antibiotic development. Science 321, 1078-1080 (2008) FURTHER READING Lock, R. L. \& Harry, E. J. Cell-division inhibitors: new insights for future antibiotics. Nature Rev. Drug Discov. 7, 324-338 (2008) 